\title{
THE COMPRESSIBILITIES OF HELIUM AND NEON.
}

\author{
BY F. P. BURT.
}

(A Paper read bcfore the Faraday Society, Tuesday, Fanuary I8, I910, Dr. F. Mollwo Perkins, Treasurer, in the Chair.)

[Communicated by Dr. N. T. M. Wilsmore.]

The determination of the $p v$ isothermals at $0^{\circ} \mathrm{C}$. of oxygen and hydrogen chloride formed part of an investigation carried out by Dr. Gray and myself on the atomic weight of chlorine (F. C. S. Transactions, I909, 95, 1657). The results obtained were satisfactory in spite of the fact that neither of these gases is particularly suitable for accurate compressibility measurements. For some unexplained reason no investigator has obtained very concordant results with oxygen, whilst the extremely hygroscopic nature of hydrogen chloride and its property of being adsorbed by glass surfaces make it a difficult gas to experiment with.

The rare gases of the atmosphere, because of their chemical inertness and low boiling-point, should afford peculiarly suitable material for such measurements, and since the lie of their isothermals is a matter of considerable interest it was decided to investigate them in the same apparatus. The compressibility of helium at $0^{\circ} \mathrm{C}$. between $\mathrm{I}$ and $\mathrm{o}$ atmospheres has been measured by Jaquerod and Scheuer (Recherches Physico-chimiques sur les Gaz., Mémoires de la Société de Physique et d'Histoire Naturelle de Genève, 1908, 35,668 ). They obtained a coefficient 0.00048 , resembling that of hydrogen, but they found that their gas contained some impurity which they concluded to be argon. Ramsay and Travers (Phil. Trans., I901, I97A., 79) made pv measurements of helium and neon between pressures of $2 \mathrm{I}$ and 77 metres of mercury, and Kammerling Onnes has recently studied the behaviour of helium between 20 and 60 atmospheres.

I was able to obtain helium and neon in sufficient quantity and in a high state of purity. The helium, which was kindly supplied by Sir William Ramsay, was made from thorianite and after passage over hot copper oxide was treated with charcoal cooled in liquid air. Spectroscopic examination showed no detectable impurity in the resulting gas. The neon was separated from a mixture of neon, helium, argon, hydrogen and nitrogen by passage over heated magnesium, calcium, and copper oxide, followed by repeated fractionation from cocoanut charcoal cooled in liquid air. With the exception of helium the gas so treated contained no impurities; its density was found to be 0.897 at $0^{\circ} \mathrm{C}$., corresponding to the presence of $0^{\circ} 5$ per cent. of helium, a quantity which was hardly likely to affect the compressibility results.

The compressibility apparatus (Fig. I) consisted essentially of a bulb $A$ connected by means of capillary tubing with a previously calibrated deadspace $B$, and a manometer $C$, both enclosed in a water-bath. The effective volume of the bulb $A$ could be altered by the introduction or withdrawal of 
mercury at the $\operatorname{tap} D$. Gas could enter the dead-space and bulb through the side tube $G$. The pressure of the gas in the bulb was obtained by measuring the distance of the upper meniscus in the manometer column above the lower meniscus set to a glass point in the dead-space. A millimetre glass

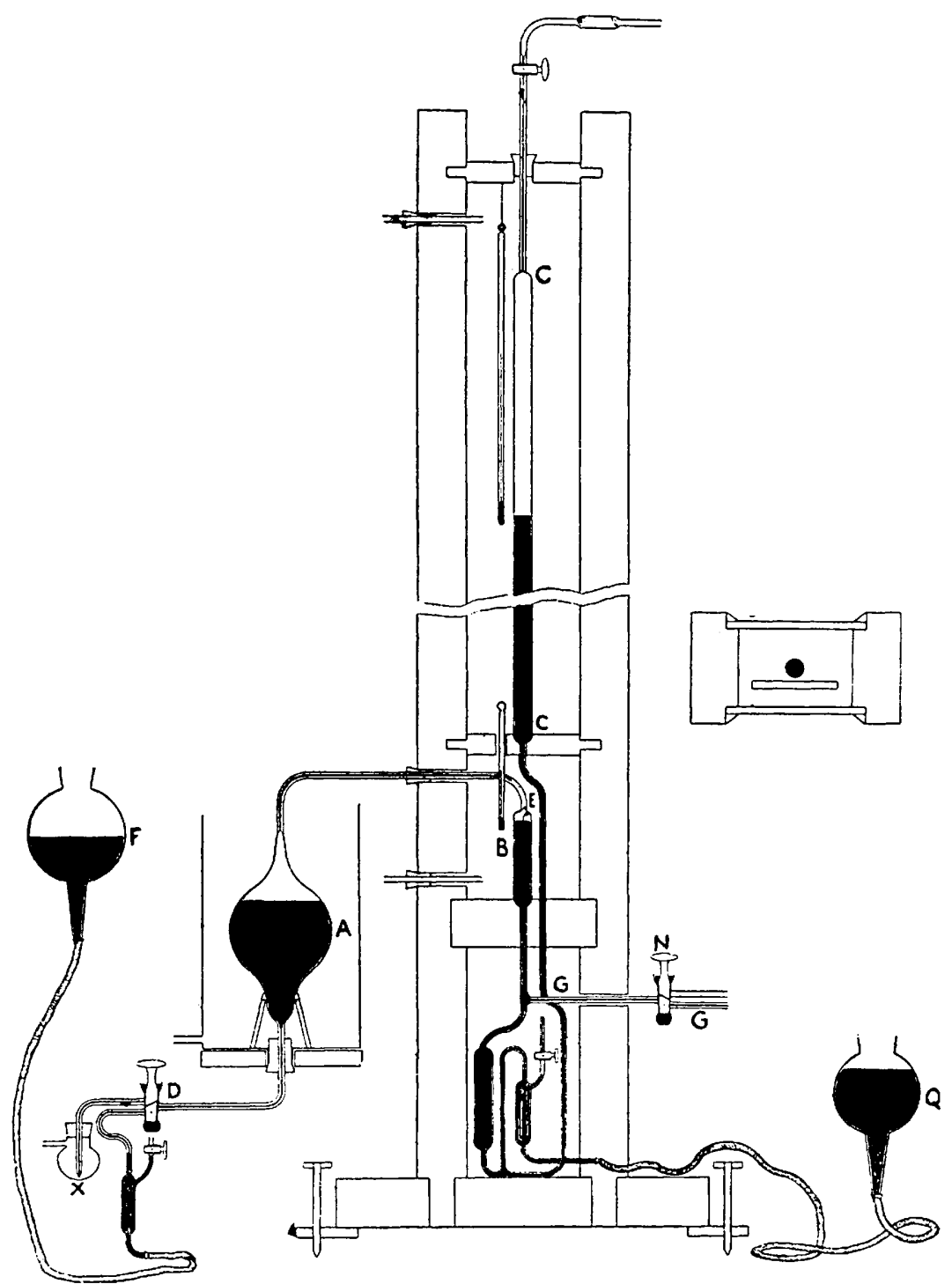

Fig. I.

scale was suspended inside the bath immediately in front of the manometer column and a telescope fitted with a Hilger micrometer eyepiece was used for determining fractions of a millimeter. All the measurements were carried out with the bulb $A$ cooled to $0^{\circ} \mathrm{C}$, in a bath of ice and water. A 
fuller description of the apparatus and of the corrections to be applied to the experimentally determined volume and pressure values may be found in the Chemical Society paper already referred to. The tables in this paper give the final values with all corrections made.

Before the introduction of the gas to be examined, the bulb $A$, which had last contained hydrogen chloride, was washed out with dry air, exhausted with a mercury pump, and finally exposed to charcoal cooled in liquid air for five or six hours. The exhaustion was carried out through the tap $N$, the mercury in the dead-space being taken below the point of entry of the

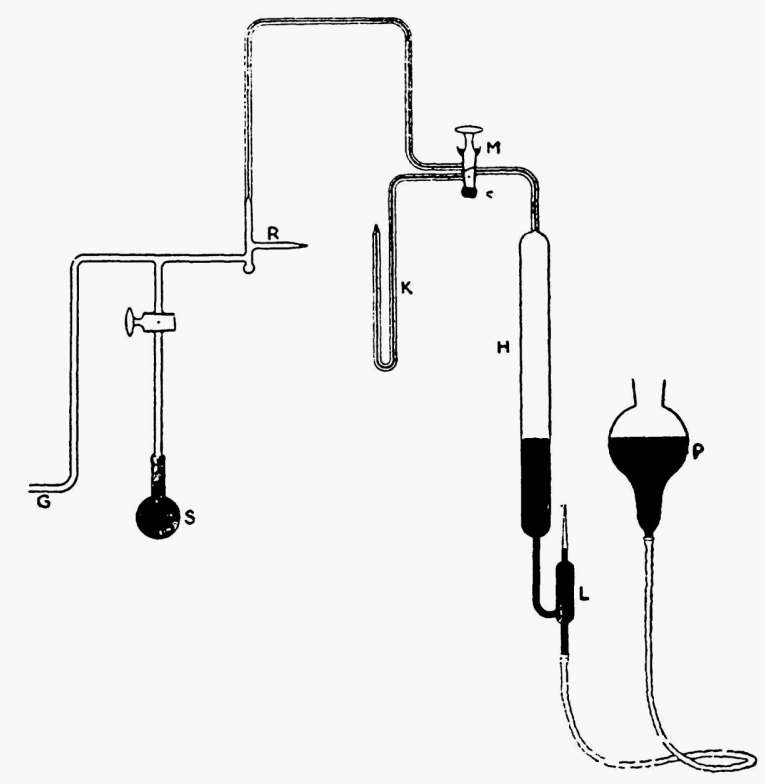

FIG. 2.

side-tube $G$. Clean, dry mercury was then allowed to flow into the vacuous bulb from the reservoir $F$ until the thread stood in the capillary in the neighbourhood of the mark $E$, just above the dead-space. At this stage the compressibility apparatus was connected with the gas-holder containing the helium or neon (Fig. 2) by sealing together the tubes $G, G^{\prime}$. The pure, dry gas was introduced into the cylinder $H$ through the capillary U-tube $K$ in the usual way. An air-catch $L$ protected the cylinder from any leakage at the rubber junction. The space between the two two-way taps, $M$ and $N$, was then exhausted by means of a pump connected with the T-tube $R$, which was afterwards sealed off. The last traces of gas or moisture were removed by the charcoal bulb $S$, which was cooled in liquid air. The upper bore of the $\operatorname{tap} M$ was exhausted or filled with mercury before the apparatus was set up.

By a suitable manipulation of the reservoirs $P$ and $Q$ and the taps $M$ and $N$ gas was admitted into the dead-space to atmospheric pressure. The mercury meniscus in the capillary was then set exactly to the mark $E$ above the dead-space by means of the tap $D . *$ Mercury was next run out of the

* A correction had to be made for the variation of the volume of the bulb as the internal pressure varied. This was previously determined and the results expressed graphically so that the correction for any pressure could be read from a curve. 
bulb $A$ into a weighed bottle $X$ until the effective volume of the bulb was of the required magnitude. The quantity of gas in the bulb and dead-space was afterwards adjusted so that when the mercury meniscus was set to the glass. point the pressure was of the order desired. As soon as the initial pressure

NEON.

\begin{tabular}{|c|c|c|c|c|}
\hline & $p$ (in $\mathrm{mm}$.). & $v$ (in c.c.). & $p v$. & Smoothed $p v$ \\
\hline I & $860^{\circ} \cdot 44$ & $68 \cdot 502$ & 58,942 & 58,944 \\
\hline 2 & $8 \mathrm{II} \cdot 58$ & $72 \cdot 626$ & 58,942 & $58,94 \mathrm{r}$ \\
\hline 3 & $775^{\circ} \cdot 64$ & $75^{\circ} 987$ & 58,939 & 58,938 \\
\hline 4 & $740 \cdot 87$ & $79^{\circ} 547$ & 58,934 & 58,935 \\
\hline 5 & $7^{22} 31$ & 81589 & 58,933 & 58,934 \\
\hline 6 & $703.5 \mathrm{I}$ & $83^{\circ} 770$ & 58,933 & $58,93^{2}$ \\
\hline 7 & 618.65 & 95.250 & 58,926 & 58,926 \\
\hline 8 & $592^{\circ} 5 \mathrm{I}$ & $99^{\circ} 449$ & 58,925 & 58,923 \\
\hline 9 & $564^{\circ} 00$ & I0 4.467 & 58,919 & 58,920 \\
\hline IO & $533^{\circ} 95$ & I IO 342 & 58,917 & 58,918 \\
\hline II & 483.59 & I 21.835 & 58,918 & 58,914 \\
\hline I2 & 438.30 & 134.408 & $58,9 \mathrm{II}$ & 58,910 \\
\hline I3 & $397^{\circ} 92$ & I $480^{\circ} \circ 3^{6}$ & 58,906 & 58,907 \\
\hline 14 & $36 \mathrm{r} \cdot 69$ & 162.850 & $58,90 \mathrm{II}$ & 58,904 \\
\hline 15 & 325.45 & I $80^{\circ} 977$ & 58,899 & $58,90 \mathrm{I}$ \\
\hline 16 & 288.69 & 204 이 5 & 58,897 & 58,897 \\
\hline I 7 & $25^{\circ} 77$ & $227^{\circ} 599$ & 58,896 & 58,895 \\
\hline I8 & $23 \times 67$ & $254^{\circ} 218$ & 58,895 & 58,893 \\
\hline I9 & 200.50 & 293.762 & 58,899 & $58,89 \mathrm{I}$ \\
\hline 20 & $170^{\circ} 29$ & $345.87 \mathrm{I}$ & 58,898 & 58,888 \\
\hline $2 \mathrm{I}$ & $144^{\circ} 4^{2}$ & 407.848 & 58,900 & 58,886 \\
\hline
\end{tabular}

HELIUM.

\begin{tabular}{|c|c|c|c|c|}
\hline & $p$ (in $\mathrm{mm}$.). & $v$ (in c.c.). & $p i$ & Smoothed pv. \\
\hline I & $837^{\circ} 63$ & $67 \cdot 547$ & 56,579 & 56,580 \\
\hline 2 & $794 \cdot 8 \mathrm{r}$ & $7 \mathrm{I} \cdot \mathrm{I} 9 \mathrm{I}$ & 56,583 & 56,580 \\
\hline 3 & $76 \mathrm{r} \cdot 56$ & $74 \cdot 293$ & 56,579 & 56,580 \\
\hline 4 & $732 \cdot 17$ & $77 \cdot 278$ & $56,58 \mathrm{I}$ & 56,580 \\
\hline 5 & 613.09 & $92 \cdot 279$ & 56,575 & 56,580 \\
\hline 6 & $56 \mathrm{r} \cdot 40$ & I00.777 & 56,576 & 56,580 \\
\hline 7 & $520: 37$ & 108.720 & 56,575 & 56,580 \\
\hline 8 & $462 \cdot 54$ & $\mathrm{I} 22 \cdot 320$ & 56,578 & 56,580 \\
\hline 9 & $409 \cdot 38$ & $138 \cdot 217$ & 56,583 & 56,580 \\
\hline Ió & $35^{8} \cdot 73$ & I $57^{\circ} 720$ & 56,579 & 56,580 \\
\hline II & $3 \mathrm{IO} \cdot 3 \mathrm{I}$ & $182 \cdot 341$ & 56,582 & 56,580 \\
\hline 12 & $269.8 \mathrm{I}$ & $209 \cdot 724$ & 56,586 & 56,580 \\
\hline I3 & 237.84 & 237.895 & $56,58 \mathrm{I}$ & 56,580 \\
\hline 14 & $199^{\circ} 6 \mathrm{I}$ & 283472 & 56,584 & 56,580 \\
\hline 15 & I69.48 & $333.88 \mathrm{I}$ & 56,586 & $56,5^{80}$ \\
\hline 16 & 147.16 & 384539 & 56,589 & 56,580 \\
\hline
\end{tabular}

of the imprisoned gas had been read a further quantity of mercury was run out, the meniscus adjusted again to the dead-space point, and the new pressure determined. When the pressure in the bulb fell to such a point that mercury would no longer flow from the tap $D$, a filter pump was attached to the side-tube of the bottle $X$. The volume increments were determined 
by weighing the successive quantities of mercury run out and the products of the total volume and corresponding pressure at each stage were plotted

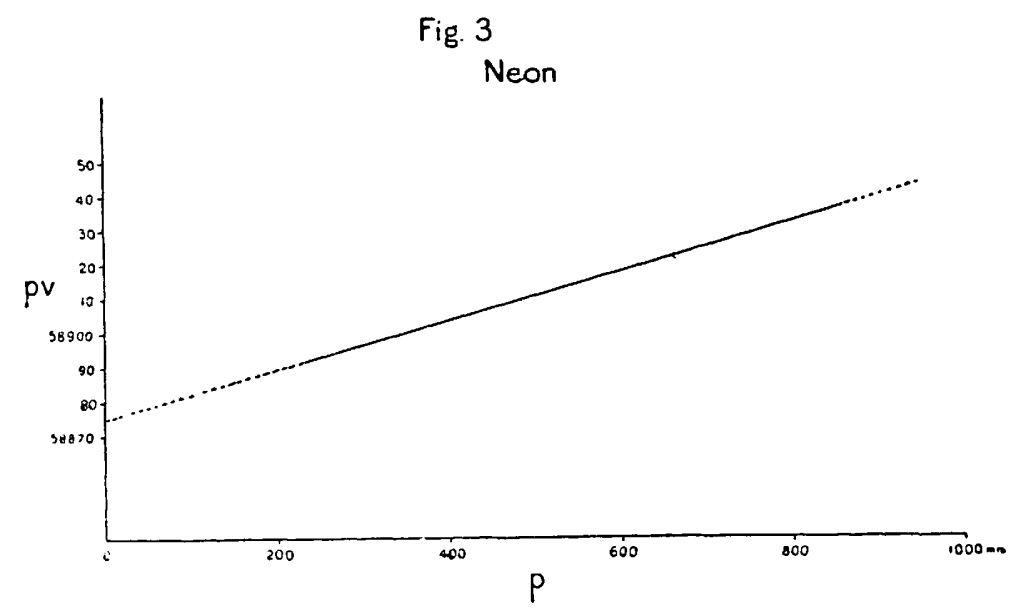

against the pressures. At the end of the following tables a full calculation of a single $p v$ value for neon (No. I7) is appended. Figs. 3 and 4 represent diagrammatically the lie of the neon and helium isothermals.

\section{Calculation of Neon $p v$, No. i7.}

Volume of Gas.

Volume of gas in bulb at previous reading, all corrections made $\begin{array}{llll}\text { except dead-space and contraction correction } & \ldots & \ldots & 202.822\end{array}$

Weight of mercury run out, $320.609 \mathrm{gm}$.

Correction for air displacement of mercury, $+0.028 \mathrm{gm}$.

Increase in effective volume of bulb, $\frac{320^{\circ} 64}{13.5955} \quad \ldots \quad \ldots \quad \ldots=23^{\circ} 5^{8} 4$

Volume of dead-space, I.275 c.c.

Temperature of gas in dead-space, $144^{\circ} \mathrm{C}$.

Volume of gas in dead-space at $0^{\circ} \mathrm{C} ., \frac{\mathrm{r} 275 \times 273}{287^{\circ} 4} \ldots \quad \ldots \quad \ldots=\mathrm{I} \cdot 2 \mathrm{rr}$

Contraction of volume of bulb under diminished internal

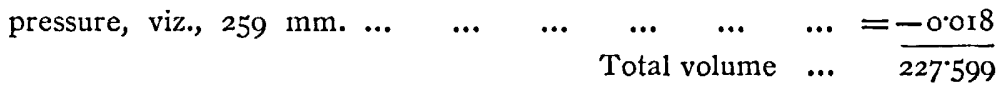

\section{Pressure of Gas.}

Distance from mercury

Micrometer Readings. Millimetre. Scale Readings.

meniscus set to point

in dead-space to scale

line 5 above it $\quad \ldots 70,7 \mathrm{I}, 70,70,69 \quad \ldots \quad 0.55 \quad \ldots \quad 5^{\circ} 00-0^{\circ} 55=4.45$ mean 70

( $\mathrm{r} 28$ micro-divs. $=\mathrm{x} \mathrm{mm}$.) 
Distance of upper menis-

cus below scale-line

$264 \quad \ldots \quad \ldots \quad \ldots$

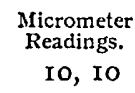

Uncorrected manometric height, $263^{\circ} 9^{2}-4^{*} 45$

Correction for total error in scale, $\frac{0.044 \times 259}{960}$

Millimetre. Scale Readings. ... $0.08 \quad \ldots 264-0.08=263^{\circ} \cdot 92$

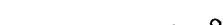

Correction for contraction of scale from $16^{\circ}$ C. to

$$
\mathrm{r} 4^{\circ} 45^{\circ} \mathrm{C} .=\mathrm{r}^{\circ} 55^{\circ} \mathrm{C} .=0.000009 \times \mathrm{I}^{\circ} 55 \times 259 \quad \ldots \quad \ldots=-0^{\circ} \quad .004
$$

Correction of observed manometric height from $144^{\circ} 45^{\circ} \mathrm{C}$.

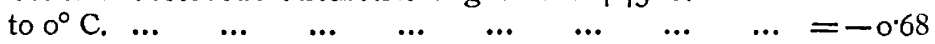

$$
\begin{aligned}
& \text { Corrected pressure } \quad \ldots=25^{\circ} 77 \mathrm{~mm} \text {. } \\
& \text { Therefore } p v=25^{8} \cdot 77 \times 227^{\circ} 599=5^{8,896} \text {. }
\end{aligned}
$$

In the case of both gases a straight line could be drawn through the plotted points, the deviations being well within the limits of experimental error. In the case of neon the agreement between the observed and smoothed values of $p v$ was surprisingly good, the greatest discrepancyneglecting the last three values-being less than $\mathrm{r}$ part in 14,000 .

It will be noted that the last three values of the neon series and the last one or two of the helium show a decided jump in the isothermal. The fact that this occurs at about the same pressure in both cases, and that a correction of about the same order would bring both sets of points into line suggests

Fig 4

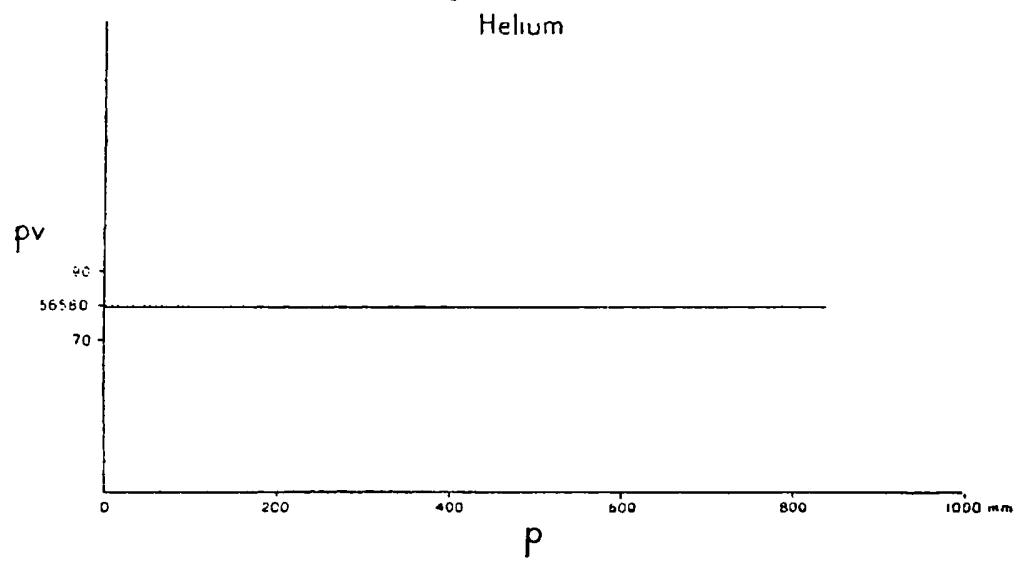

that the phenomenon is due to some constant error which conceals the true form of the isothermal in this region. It is just possible that there was some slight distortion in the manometer tube, as these readings were made not far above the point where the glass was drawn out (see Fig. I). For these reasons, in extrapolating to zero pressure to find the value of $p_{0} v_{0}$ for neon, the last three points have been given no weight.

The values of $p_{0} v_{0}$ and $p_{\mathrm{x}} v_{\mathrm{x}}$ were taken from the smoothed graph and found 
to be 58,875 and 58,937 respectively ; therefore the compressibility coefficient between I and o atmospheres-

$$
=\mathrm{A}_{\mathrm{O}}^{\mathrm{I}}=\frac{p_{\mathrm{x}} v_{\mathrm{x}}-p_{\mathrm{o}} v_{\mathrm{o}}}{p_{\mathrm{o}} v_{\mathrm{o}}\left(p_{\mathrm{x}}-p_{\mathrm{o}}\right)}=\frac{5^{8,937-58,875}}{5^{8,875}}=+0.00105
$$

Neon, like hydrogen, is plus que parfait at $0^{\circ} \mathrm{C}$., but the coefficient is nearly twice as great.

The lie of the helium isothermal is rather astonishing. The only possible straight line that can be drawn through the points is one parallel to the pressure axis, so that the compressibility coefficient between $\mathrm{I}$ and $\mathrm{O}$ atmospheres is apparently $o$. From the critical temperature one would have expected the isothermal at $0^{\circ} \mathrm{C}$. to slope towards the pressure axis as the pressure diminished like those of hydrogen and neon. The comparatively large number of readings (sixteen) and their satisfactory agreement makes one disinclined to suspect experimental errors, and further, it is extremely improbable that any constant error would affect the values in such a way as to make them all the same. The helium was carefully examined again after the measurements had been made, and no trace of impurity could be detected spectroscopically. Experimental determination of helium isothermals at temperatures above and below $0^{\circ} \mathrm{C}$. would be interesting, and might establish the correctness or incorrectness of the above results.

In conclusion, I wish to thank Professor Collie and Mr. H. E. Watson for their kindness in placing at my disposal specimens of the two gases which they had recently purified in quantity for density determinations.

University College, LONDON. 